\title{
Dual roles of WISP2 in the progression of hepatocellular carcinoma: implications of the fibroblast infiltration into the tumor microenvironment
}

\author{
Qingan Jia ${ }^{1}$, Yaoyao Zhang ${ }^{1}$, Binghui $\mathrm{Xu}^{1}, \mathrm{Xia} \mathrm{Liao}^{2}$, Yang Bu${ }^{3}$, Zihan $\mathrm{Xu}^{4}$, Xianglong Duan ${ }^{5}$, \\ Qiangbo Zhang ${ }^{6,7}$ \\ ${ }^{1}$ Institute of Medical Research, Northwestern Polytechnical University, Xi'an 710072, China \\ ${ }^{2}$ Department of Nutrition, The First Affiliated Hospital of Xi' an Jiaotong University, Xi'an 710061, China \\ ${ }^{3}$ Department of Hepatobiliary Surgery, General Hospital, Ningxia Medical University, Yinchuan 750001, China \\ ${ }^{4}$ Department of Burns and Plastic Surgery, Affiliated Shaanxi Provincial People's Hospital, Northwestern \\ Polytechnical University, Xi'an 710068, China \\ ${ }^{5}$ Second Department of General Surgery, Shaanxi Provincial People's Hospital Affiliated Hospital of Northwestern \\ Polytechnical University, Xi'an 710068, China \\ ${ }^{6}$ Cheeloo College of Medicine, Shandong University, Jinan 250012, China \\ ${ }^{7}$ Department of General Surgery, Qilu Hospital, Shandong University, Jinan 250012, China
}

Correspondence to: Qiangbo Zhang; email: 201362006584@email.sdu.edu.cn

Keywords: HCC, WISP2, HMGB1, fibroblast, prognosis

Received: April 8, $2021 \quad$ Accepted: July 8, $2021 \quad$ Published: September 8, 2021

Copyright: (C) 2021 Jia et al. This is an open access article distributed under the terms of the Creative Commons Attribution License (CC BY 3.0), which permits unrestricted use, distribution, and reproduction in any medium, provided the original author and source are credited.

\section{ABSTRACT}

The dismal outcome of hepatocellular carcinoma (HCC) patients is attributable to high frequency of metastasis and. Identification of effective biomarkers is a key strategy to inform prognosis and improve survival. Previous studies reported inconsistent roles of WISP2 in carcinogenesis, while the role of WISP2 in HCC progression also remains unclear. In this study, we confirmed that WISP2 was downregulated in HCC tissues, and WISP2 was acting as a protective factor, especially in patients without alcohol intake using multiple online datasets. In addition, we reported that upregulation of WISP2 in HCC was related to inhibition of the malignant phenotype in vitro, but these alterations were not observed in vivo. WISP2 also negatively correlated with tumour purity, and increased infiltration of fibroblasts promoted malignant progression in HCC tissues. The enhanced infiltration ability of fibroblasts was related to upregulated HMGB1 after overexpression of WISP2 in HCC. The findings shed light on the anticancer role of WISP2, and HMGB1 is one of the key factors involved in the inhibition of the efficiency of WISP2 through reducing the tumour purity with fibroblast infiltration.

\section{INTRODUCTION}

Hepatocellular carcinoma (HCC) has high incidence rates in China, which accounts for more than $50 \%$ of the total number of liver cancer cases and deaths in the world [1]. Even though many advanced strategies, including liver transplantation, molecular targeted therapies and immune-based treatments, the general prognosis of patients with $\mathrm{HCC}$ is still unsatisfactory [2]. Therefore, continued identification of new molecules for the development of combining targeted therapy is still urgently needed.

Cellular communication network $(\mathrm{CCN})$ family are scaffolding proteins that may govern and balance the interconnection among individual signaling pathways. 
$\mathrm{CCN}$ proteins are a six-member family of cysteine-rich regulatory proteins that exist only in vertebrates, including CCN1 (cysteine-rich 61, CYR61), CCN2 (connective tissue growth factor, CTGF), CCN3 (nephroblastoma overexpressed, NOV), CCN4 (Wnt1inducible signaling pathway proteins, WISP-1), CCN5 (WISP-2), and CCN6 (WISP-3) [3]. The diverse effects of physiological and pathological events are attributed to each structural domain of CCNs (with CCN5 lacking the CT domain): IGFBP, VWC, TSP-1, and CT [4]. Although CCN family members have highly consistent biological structures, these factors have differential expression and play specific roles in biology in different human cancers [5]. WISP2 differs from the other CCN family members due to a lack of the C-terminal domain, which has been shown to interact with extracellular cytokines, and receptors such as, integrins, EGFR, Notch 1 and LRP6 [6]. WISP2 can also interfere with the other $\mathrm{CCN}$ isoforms, influencing the function of these protein family members [7]. The expression and function of WISP2 are diverse in different human cancers. In breast cancer, WISP2 was reported as an oncogenic role [8]. By contrast, WISP2 is downregulated in human leiomyoma [9], pancreatic adenocarcinoma [10], salivary gland cancer [10], colorectal tumors [11], and gallbladder cancer [12], suggesting that it acts as a tumor suppressor. Up to now, the role of WISP2 in tumor progression also remains unclear in HCC [13].

In the present study, we analysed expression of WISP2 and the prognostic correlation in HCC patients using Oncomine, Kaplan-Meier plotter, and Gene expression profiling interactive analysis 2 (GEPIA2). We explored the role of WISP2 in HCC using the Cancer Cell Line Encyclopedia (CCLE) and gene microarrays and then assessed the correlation between WISP2 and stromal cells in tumour tissues using the Tumor IMmune Estimation Resource (TIMER). Finally, the role of WISP2 and its relationship with tumour purity and fibroblast infiltration were examined both in vitro and in vivo. Our studies revealed the anticancer role for WISP2 was conditional in HCC, and the efficiency was influenced by fibroblast infiltration in the tumor microenvironment (TME).

\section{MATERIALS AND METHODS}

mRNA expression, gene correlation, tumour purity, and immune infiltrate analysis in TIMER

TIMER is a comprehensive resource for analysis of immune infiltrates across diverse cancer types (https://cistrome.shinyapps.io/timer/) [14]. The expression levels of WISP2 between tumour and adjacent normal tissues in different types of cancer were identified across all TCGA tumours via 'Diff Exp' module. The correlation between WISP2 expression and immune infiltration, including $\mathrm{B}$ cells, $\mathrm{CD}^{+}{ }^{+} \mathrm{T}$ cells, $\mathrm{CD} 8^{+} \mathrm{T}$ cells, neutrophils, macrophages, and dendritic cells, as well as a pairwise gene (CD34, PECAM, VCAM1, NT5E, ESM1, S100A4, VIM, and ACTA2) correlation in liver cancer were explored via 'Gene' and 'Correlation' modules, respectively.

\section{mRNA expression and survival analysis in GEPIA2}

GEPIA2 is resource for analyzing the RNA sequencing expression data of 9,736 tumours and 8,587 normal samples from the TCGA and the GTEx projects [15]. WISP2 tumour/normal differential expression and the correlation between WISP2 expression and survival in diverse cancer types are analysed in GEPIA2.

\section{mRNA expression in cancer cell lines in the CCLE}

WISP2 expression levels in cancer cell lines from diverse cancer types were examined using the CCLE (http://www.broadinstitute.org/ccle), which provides public access to genomic data, analysis, and visualization for more than 1,100 cell lines [16].

\section{Survival analysis in Kaplan-Meier plotter}

The correlation between WISP2 expression and survival in liver cancer was analysed using the Kaplan-Meier plotter (http://kmplot.com/analysis/) [17]. The KaplanMeier plotter can assess the effects of 54,000 genes on survival in 21 cancer types. Gene expression data and RFS and OS information were downloaded from GEO, EGA, and TCGA.

\section{Protein-protein interaction (PPI) analysis in GeneMANIA}

GeneMANIA is an online tool that predicts the function of genes and gene sets, including protein and genetic interactions, pathways, co-expression, co-localization, and protein domain similarity, in GeneMANIA [18] (https://genemania.org). WISP2 and CTGF were used as queries to predict PPIs, and the prediction output graphically shows a network that depicts the relationships between genes in the list.

\section{Clinical features analysis in LinkedOmics database}

The relationship between WISP2 expression and clinical features in liver cancer patients was analysed using the LinkedOmics database (http://www.linkedomics.org), which is a publicly available portal that includes 
multi-omics data from all 32 TCGA Cancer types [19]. Based on the platform, a statistical analysis of the correlation between WISP2 expression and clinical features of $\mathrm{HCC}$ was performed.

\section{cDNA microarray analysis}

cDNA microarrays were performed using the Human OneArray ${ }^{\circledR}$ (Phalanx Biotech Group, San Diego, CA, USA) to evaluate the alterations of expression profiling. Total RNA was extracted from Hep3B-WISP2 and Hep3B-Vector cells and the isolations and microarray analyses were performed in triplicate according to the manufacturer's instructions. All data was uploaded to the Gene Expression Omnibus (GSE134563).

Patients and tissue microarray analysis, cell lines culture, vector construction and transfection, various functional assays of WISP2 in vivo (animal models with subcutaneous xenografts) and in vitro (migration, invasion, and proliferation) were all exhibited in Supplementary Materials.

\section{Statistical analysis}

Graphics were drawn using GraphPad Prism version 6 (GraphPad Software, La Jolla, CA, USA). Statistical analyses were performed using SPSS 15.0 for Windows (SPSS). A $p$-value $<0.05$ was considered statistically significant.

\section{Ethics approval}

Animal protocols were approved by the Medical Experimental Animal Care Commission of Northwest Polytechnical University, and all methods were performed in accordance with the relevant guidelines.

\section{RESULTS}

\section{WISP2 mRNA levels was lower in tumour tissues of HCC compared with normal tissues, but the antitumor role of WISP2 is conditional}

Using the TIMER and GEPIA2 database to evaluate the RNA-seq data, expression of WISP2 was found to be significantly downregulated in most human cancers, including HCC, compared with the associated normal tissues. Only in kidney renal clear cell carcinoma (KIRC) and kidney renal papillary cell carcinoma (KIRP) were WISP2 expression significantly higher than that in adjacent normal tissues (Figure 1A, 1B). To explore the role of WISP2 on prognosis, 33 human cancers were included using the GEPIA2 database. The role of WISP2 on prognosis varied in different types of cancers. In HCC, WISP2 had a protective role, as high expression was associated with better prognosis for this cancer type, although there was no significant statistical difference (Figure 1C).

To better understand the role of WISP2 in HCC prognosis, the Kaplan-Meier plotter was used. The HCC patients in the WISP2-high group had longer OS than the WISP2-low group, although there was no significant difference $(\mathrm{HR}=0.7, p=0.067)$. The patients in the WISP2-high group had a significantly longer RFS compared with those in the WISP2-low group (HR $=0.62, p=0.011$; Figure $1 \mathrm{D})$. These results revealed WISP2 was acting as a protective factor and the level of WISP2 was decreased in HCC.

We evaluated the effects of clinicopathologic characteristics on the prognosis of HCC patients with different WISP2 expression levels. High expression of WISP2 was associated with longer OS in female patients $(\mathrm{HR}=0.52, p=0.026)$. Specifically, high expression of WISP2 mRNA was correlated with longer OS in stage $2(\mathrm{HR}=0.37, p=0.01)$ and longer RFS in stage $1(\mathrm{HR}=0.5, p=0.013)$ in HCC patients. High WISP2 expression was correlated with longer OS in grade $1(\mathrm{HR}=0.3, p=0.023)$ and $3(\mathrm{HR}=0.45, p=$ $0.017)$ patients. In addition, high WISP 2 expression was correlated with longer RFS in patients with no vascular invasion ( $\mathrm{HR}=0.57, p=0.028), \mathrm{T} 1(\mathrm{HR}=0.49, p=$ $0.0078)$, and $\mathrm{T} 2(\mathrm{HR}=0.36 p=0.018)$ according to the criteria of the American Joint Committee on Cancer (AJCC). These results suggest that WISP2 expression levels can significantly influence the prognosis in most human cancers, but the role of WISP2 in HCC is conditional (Table 1).

Upregulation of WISP2 in HCC is related to inhibited malignant phenotype in vitro

To determine the precise function of WISP2, we first evaluated the expression of WISP2 in different human tumour cell lines from the CCLE, revealing the significant differences in the expression of diverse tumors (Figure 2A). We further analysed the WISP2 expression in $25 \mathrm{HCC}$ cell lines from the CCLE, and found that the expression of WISP2 was low in $76 \%$ HCC cell lines, especially in Hep3B and HepG2 cells (Figure 2B).

In addition, we stably overexpressed WISP2 in Hep3B and HepG2 cells, and confirmed WISP2 expression using immunoblotting (Figure 2G). Hep3B-WISP2 and HepG2-WISP2 were compared with the associated control cells. Overexpression of WISP2 significantly inhibited the migration $(35.67 \pm 8.33$ vs. $66.05 \pm 12.51$, $p=0.0228)$ and invasiveness $(19.04 \pm 6.56$ vs. $37.67 \pm$ 
6.51, $p=0.0249)$ of Hep3B cell lines compared to the vector control (Figure 2C). Overexpression of WISP2 significantly inhibited the migration $(34.80 \pm 12.95$ vs. $70.12 \pm 10.02, p=0.0013)$ and invasiveness (22.81 \pm 7.86 vs. $48.25 \pm 11.82, p=0.0039)$ of HepG2 cell lines compared to the vector control (Figure 2D). We next examined the proliferative ability of these cells using a colony formation assay. Overexpression of WISP2 significantly impaired the colony formation of Hep3B cell lines ( $32.50 \pm 18.05$ vs. $128.81 \pm 29.81, p=0.0015)$ and HepG2 cell lines $(13.13 \pm 7.44$ vs. $120.81 \pm 26.86$, $p=0.0004$ ) compared to the vector control (Figure 2E). This proliferation inhibition following overexpression of WISP2 in Hep3B and HepG2 cell lines was confirmed with a CCK8 assay (Figure 2F).

We also evaluated markers related to the epithelialmesenchymal transition (vimentin, Snail, and E-cadherin) as a measure of migration and invasiveness and $\mathrm{Ki} 67$ as a proliferation marker at the protein level in HCC cells. The expression of Ki67, vimentin, and Snail were significantly downregulated, and the epithelial cell surface marker E-cadherin was upregulated in Hep3B and HepG2 cells that overexpressed WISP2 (Figure 2G). All of these results suggest that WISP2 expression is acting as a protective factor in vitro.
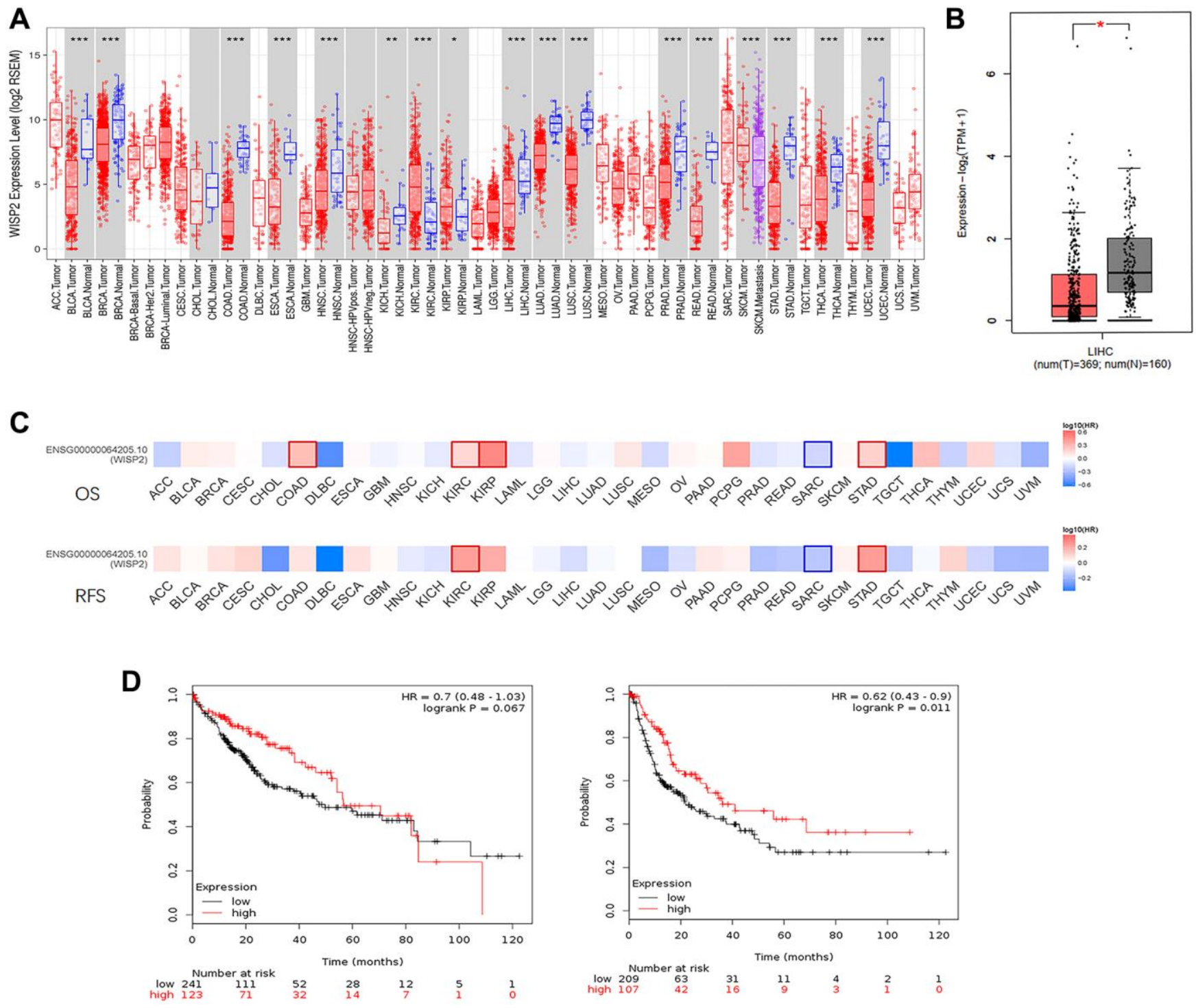

Figure 1. WISP2 mRNA level is lower compared with normal tissues, and low expression of WISP2 is associated with poor prognosis. (A) TIMER database was used to evaluate the WISP2 RNA-seq data in human cancers, and the expression of WISP2 was lower in most human cancers, including HCC. (B) The expression of WISP2 was lower in tumour tissues of HCC than normal tissues in GEPIA2 database. (C) The role of WISP2 on prognosis varied in 33 human cancers, and in HCC, WISP2 had a protective role from GEPIA2 database. (D) The protective role of WISP2 in HCC was confirmed using the Kaplan-Meier plotter, and low expression of WISP2 is associated with poor prognosis. 
Table 1. Correlation of WISP2 mRNA expression and clinical prognosis in HCC with different clinicopathological factors according to the Kaplan-Meier plotter.

\begin{tabular}{|c|c|c|c|c|c|}
\hline \multirow{2}{*}{$\begin{array}{l}\begin{array}{c}\text { Clinicopathological } \\
\text { characteristics }\end{array} \\
\operatorname{Sex}\end{array}$} & \multirow[t]{2}{*}{$\begin{array}{l}\text { No. of } \\
\text { patients }\end{array}$} & \multicolumn{2}{|c|}{$\begin{array}{c}\text { Overall survival }(\mathrm{OS}) \\
(n=364)\end{array}$} & \multicolumn{2}{|c|}{$\begin{array}{c}\text { Relapse-free survival (RFS) } \\
(n=313)\end{array}$} \\
\hline & & HR $(95 \%$ CI $)$ & $p$ & HR $(95 \%$ CI $)$ & $p$ \\
\hline Male & 250 & $0.66(0.38-1.14)$ & 0.14 & $0.58(0.37-0.91)$ & 0.016 \\
\hline Female & 121 & $0.52(0.29-0.93)$ & 0.026 & $0.52(0.25-1.09)$ & 0.077 \\
\hline \multicolumn{6}{|l|}{ Stage } \\
\hline 1 & 171 & $0.68(0.36-1.29)$ & 0.23 & $0.5(0.29-0.87)$ & 0.013 \\
\hline 2 & 86 & $0.37(0.17-0.82)$ & 0.01 & $0.21(0.03-1.76)$ & 0.12 \\
\hline $3+4$ & 90 & $0.058(0.33-1.03)$ & 0.059 & $0.48(0.23-1.02)$ & 0.051 \\
\hline \multicolumn{6}{|l|}{ Grade } \\
\hline 1 & 55 & $0.43(0.16-1.17)$ & 0.091 & $0.3(0.1-0.9)$ & 0.023 \\
\hline 2 & 177 & $0.67(0.4-1.15)$ & 0.14 & $0.75(0.46-1.23)$ & 0.26 \\
\hline 3 & 122 & $0.52(0.24-1.13)$ & 0.094 & $0.45(0.23-0.88)$ & 0.017 \\
\hline \multicolumn{6}{|l|}{ AJCC-T } \\
\hline 1 & 181 & $0.73(0.4-1.33)$ & 0.31 & $0.49(0.29-0.84)$ & 0.0078 \\
\hline 2 & 94 & $0.43(0.21-0.9)$ & 0.022 & $0.36(0.15-0.86)$ & 0.018 \\
\hline 3 & 80 & $0.59(0.32-1.08)$ & 0.083 & $1.67(0.8-3.52)$ & 0.17 \\
\hline \multicolumn{6}{|l|}{ Vascular invasion } \\
\hline none & 205 & $0.71(0.39-1.28)$ & 0.25 & $0.57(0.35-0.95)$ & 0.028 \\
\hline micro & 93 & $1.6(0.74-3.47)$ & 0.23 & $0.78(0.39-1.57)$ & 0.48 \\
\hline \multicolumn{6}{|l|}{ Alcohol intake } \\
\hline Yes & 117 & $0.53(0.25-1.12)$ & 0.089 & $0.65(0.33-1.28)$ & 0.21 \\
\hline No & 205 & $0.61(0.39-0.97)$ & 0.033 & $0.56(0.35-0.9)$ & 0.016 \\
\hline \multicolumn{6}{|l|}{ Hepatitis virus } \\
\hline Yes & 153 & $0.67(0.34-1.34)$ & 0.26 & $0.56(0.34-0.93)$ & 0.023 \\
\hline No & 169 & $0.51(0.31-0.84)$ & 0.0065 & $0.54(0.29-1.01$ & 0.052 \\
\hline
\end{tabular}

Bold text means the difference has statistical significance $(p<0.05)$.

The increased infiltration of fibroblasts plays a negative feedback role in inhibition of the efficiency of WISP2 in vivo

In this section, the role of WISP2 was evaluated in vivo. While, in nude mouse models, subcutaneous tumour growth after 4 weeks did not differ in mice injected with Hep3B-WISP2 and Hep3B-Vector $(1.23 \pm 0.15 \mathrm{~mm}$ vs. $1.33 \pm 0.12 \mathrm{~mm}, p=0.2354$; Figure 3A, 3a). And subcutaneous tumour growth also did not differ in mice injected with HepG2-WISP2 and HepG2-Vector (1.40 \pm $0.14 \mathrm{~mm}$ vs. $1.58 \pm 0.15 \mathrm{~mm}, p=0.103$; Figure $3 \mathrm{~A}, 3 \mathrm{~b}$ ). This finding was in direct contrast to our in vitro results. Additionally, we classified the patients into subgroups according to alcohol intake history, the major aetiologies of liver cancer in western countries. In patients without alcohol intake history, patients with high WISP2 expression showed significantly longer OS and RFS compared with those with low WISP2 expression (Figure 3B, 3a). While, there was no significant difference in OS and RFS between high and low WISP2 expression in patients with alcohol intake history (Figure 3B, 3b). It is now clear that alcohol consumption is closely related to liver fibrosis [20]. Thus, we sought to examine tumour microenvironmental influences on the WISP2 effects.

Stromal cells play an important role in cancer progression, and tumour-infiltrating lymphocytes are an independent predictor of survival in cancers, especially in HCC. Therefore, we first investigated whether WISP2 expression was correlated with tumour purity in HCC using TIMER database. Indeed, WISP2 expression was significantly negatively correlated with 
A
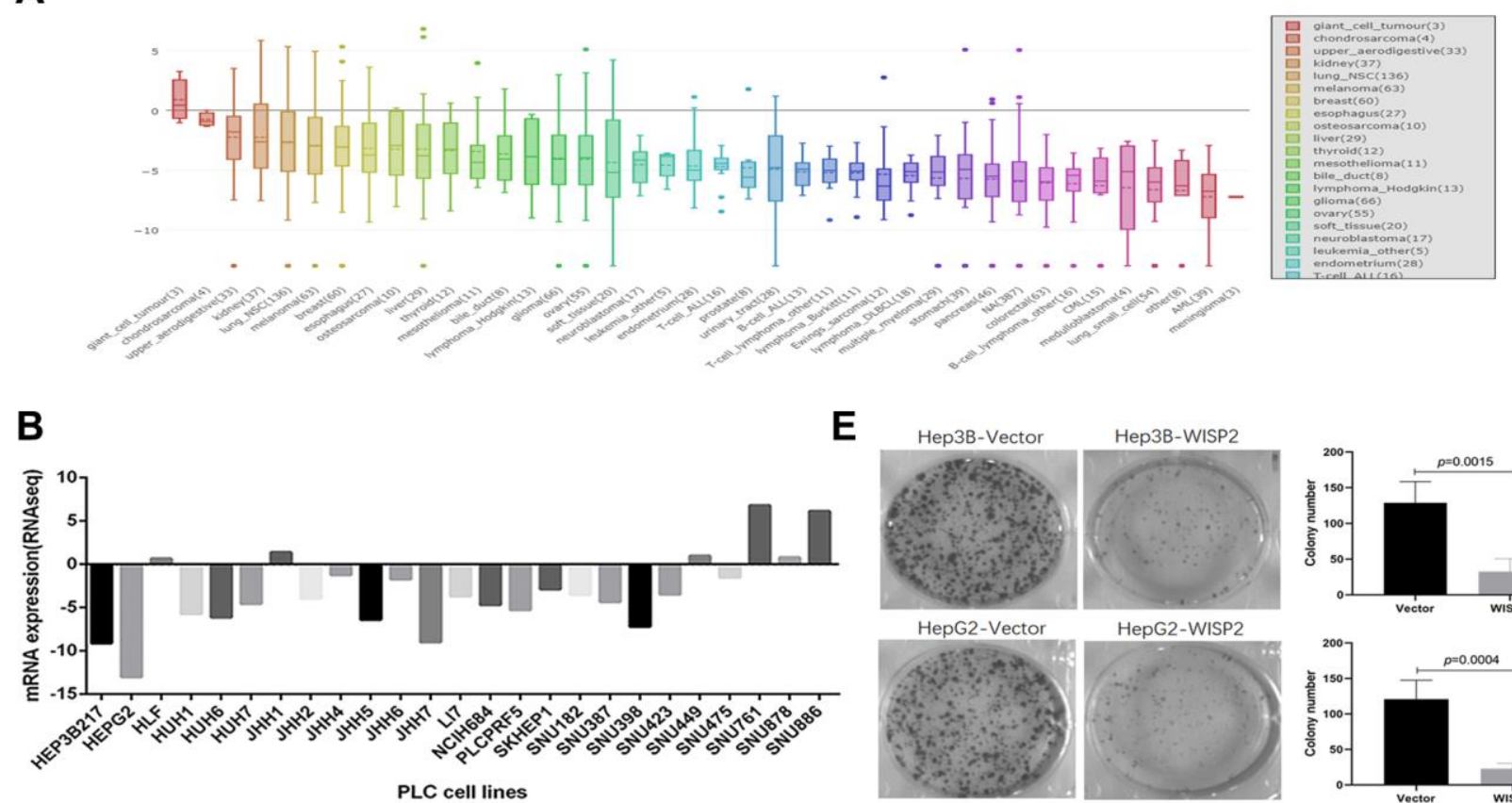

E
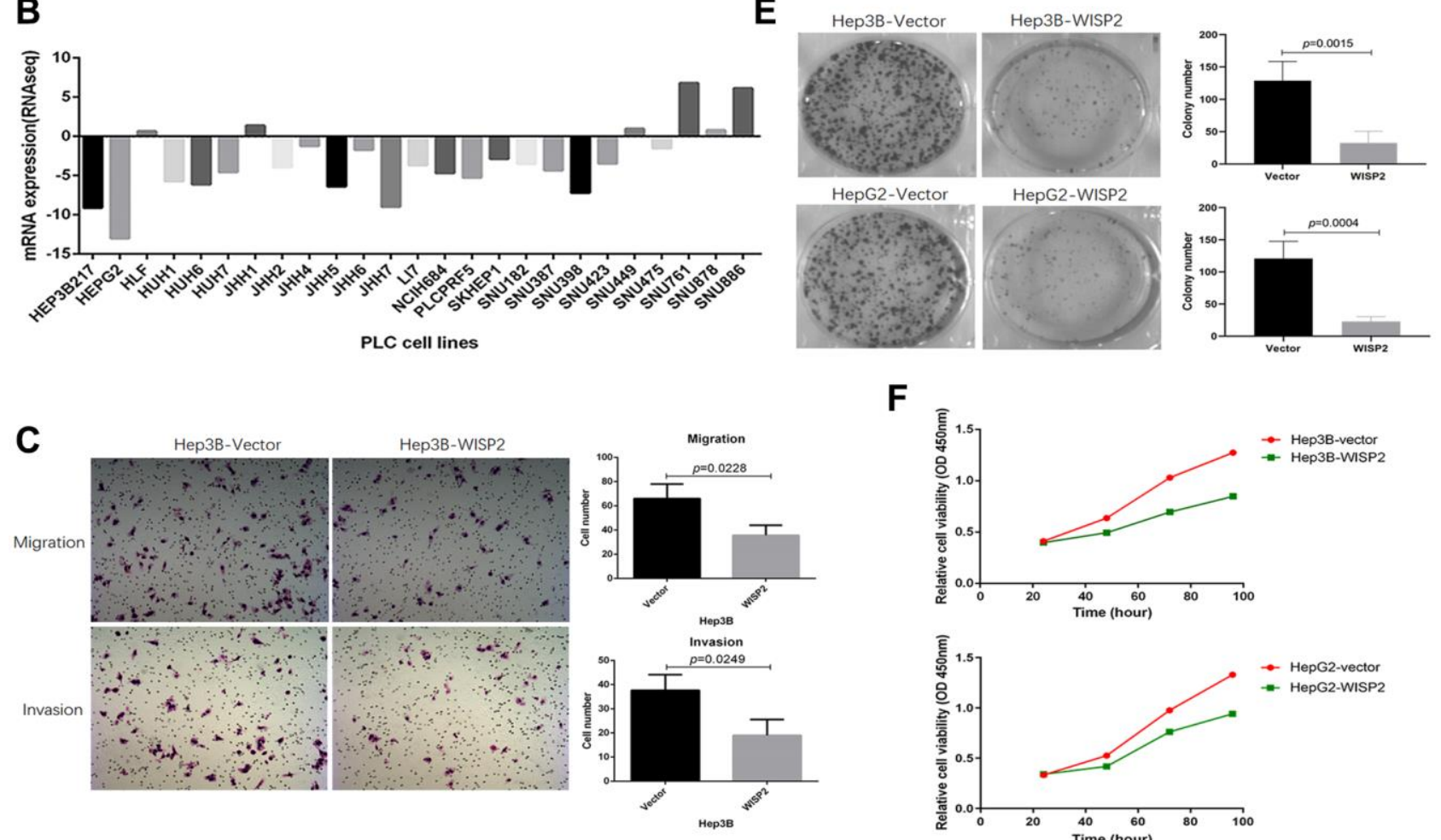

$\mathbf{F}$
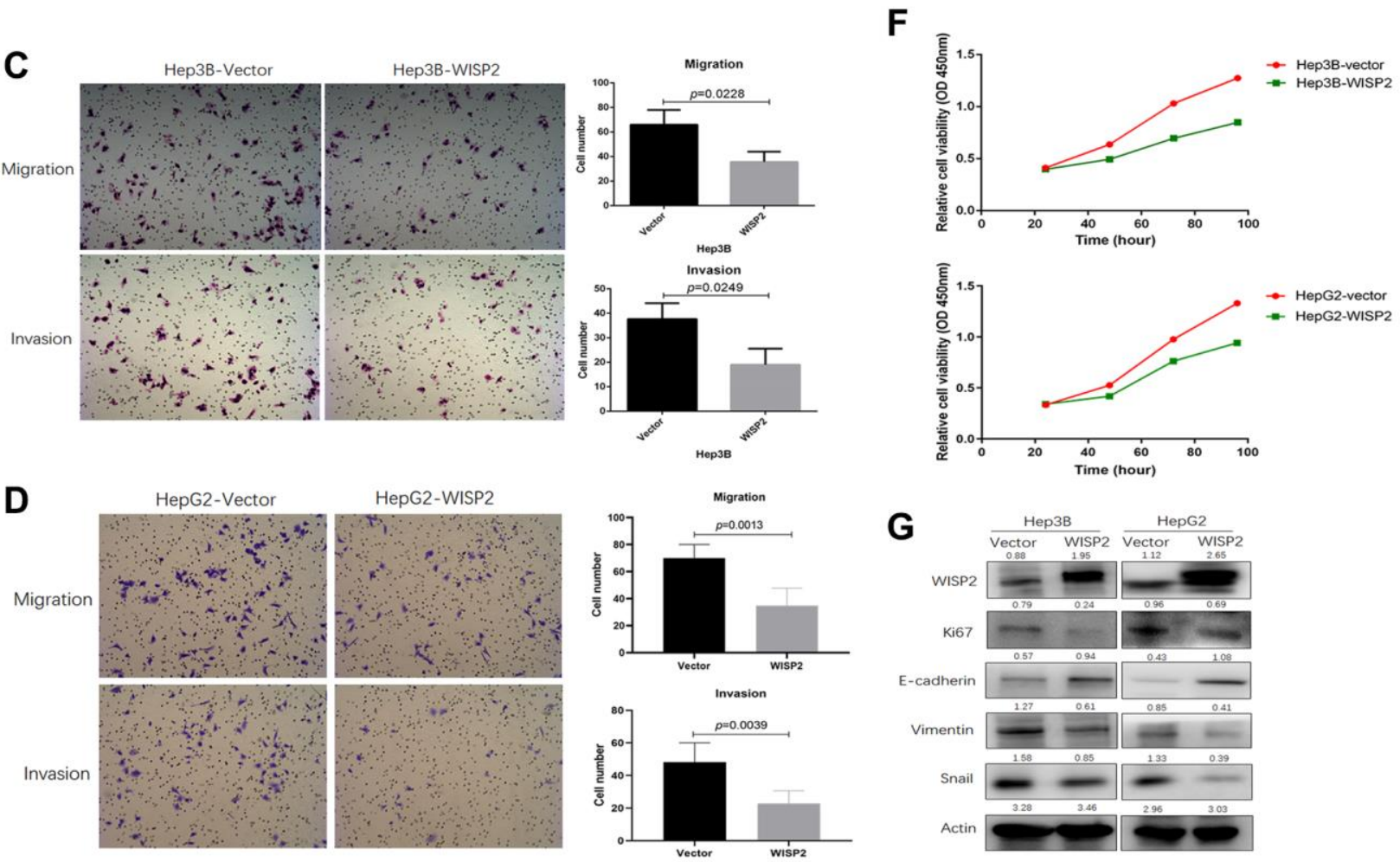

Figure 2. Upregulation of WISP2 in HCC is related to inhibition of the malignant phenotype in vitro, while no difference in proliferation was found in subcutaneous tumorigenesis in vivo. (A) The expression of WISP2 was diverse in different tumour cell lines in the CCLE database. (B) The expression of WISP2 in 76\% HCC cell lines, including Hep3B and HepG2, were low in CCLE database. (C) The expression of WISP2 was determined in Hep3B and HepG2, and confirmed using immunoblotting and immunocytochemistry. (D) Overexpression of WISP2 significantly inhibited the migration and invasiveness in Hep3B and HepG2 HCC cells. (E) Proliferation in Hep3B and HepG2 HCC cells that overexpress WISP2 or control cells was examined using a plate colony formation assay, and overexpression of WISP2 significantly impaired the colony formation. (F) Proliferation was assessed using a CCK8 assay, and overexpression of WISP2 significantly inhibited the proliferation in Hep3B and HepG2 HCC cells. (G) The expression of Ki67, vimentin, and Snail were significantly downregulated, and the epithelial cell surface marker E-cadherin was upregulated in Hep3B and HepG2 cells that overexpressed WISP2. 
tumour purity $(r=-0.517, p=5.01 \mathrm{e}-25)$, indicating that more stromal cells were present in HCC tissues with high WISP2 expression. In addition, WISP2 expression was weakly correlated with infiltrating lymphocytes, such as B cells $(r=0.068, p=2.08 \mathrm{e}-1), \mathrm{CD}^{+} \mathrm{T}$ cells $(r=0.178, p=9.37 \mathrm{e}-4), \mathrm{CD}^{+}{ }^{+} \mathrm{T}$ cells $(r=0.217$, $p=4.99 \mathrm{e}-5)$, macrophages $(r=0.181, p=7.82 \mathrm{e}-4)$, neutrophils $(r=0.113, p=3.61 \mathrm{e}-2)$, and dendritic cells $(r=0.174, p=1.31 \mathrm{e}-3$; Figure 3C, 3a). We also explored the relationship between WISP2 expression and infiltrating vascular endothelial cells and fibroblasts. Interestingly, WISP2 expression was correlated with the specific marker of vascular endothelial cells marker CD34 ( $r=0.373, p=1.03 \mathrm{e}-13)$, CD31(PECAM1, $r=$ $0.479, p=1.25 \mathrm{e}-22)$, VCAM1 $(r=0.156, p=2.54 \mathrm{e}-3)$, NT5E $(r=-0.065, p=2.15 \mathrm{e}-1)$, and ESM1 $(r=-0.34$, $p=2.54 \mathrm{e}-3$; Figure 3C, 3b). And the most relevant are WISP2 expression and the fibroblast markers S100A4 $(r=0.437, \quad p=1.03 \mathrm{e}-18)$ and $\alpha$-SMA (ACTA2; $r=0.544, p=5.3 \mathrm{e}-30$ ), and Vimentin (Vim; $r=0.579$, $p=1.34 \mathrm{e}-34$; Figure $3 \mathrm{C}, 3 \mathrm{c})$. We next examined the interactions between WISP2 and other proteins using GeneMANIA database for cancer genomics database, illuminating a strong interaction among WISP2, connective tissue growth factor (CTGF), and ACTA2 (Figure 3D). These results suggest that WISP2 expression reflects fibroblast infiltration in the tumour.

Additionally, we further examined the co-expression of WISP2 and $\alpha$-SMA in subcutaneous tumour tissues. Increased numbers of fibroblasts and fibro-collagen deposition were positive correlated with the expression of WISP2 in HCC (Figure 3E). Human liver cancer tissue microarrays confirmed a positive correlation between WISP 2 and $\alpha$-SMA (Figure 3F). These results suggested that WISP2 played a negative role in tumour purity by promoting fibroblast infiltration into the tumour microenvironment.

\section{Upregulation of WISP2 in HCC cells significantly alters gene expression profiles}

Gene expression profiles were significantly altered in Hep3B cells that overexpressed WISP2 compared to vector control cells, with 415 differentially expressed genes (Figure 4A). We analysed these altered genes using Gene Ontology (GO, Figure 4B) and Kyoto Encyclopedia of Genes and Genomes (KEGG, Figure 4C) pathway enrichment. According to GO analysis, 25 genes involved in wound healing, were significantly changed, and $80 \%$ of these were inhibited by WISP2 overexpression. The downregulated genes included KLK8, MGLL, ENO3, ALOX5, BLNK, CFI, IRAK2, GPR68, and APOL3 (Figure 4D, 4a). With respect to anti-apoptosis factors, 11 genes were significantly altered by WISP2 overexpression (Figure 4D, 4b).
Twenty five genes related to the cell cycle were significantly changed, and 8 core genes were significantly downregulated (Figure 4D, 4c). Of the 10 drug resistance-related genes that were altered, nine were downregulated by WISP2 overexpression (Figure 4D, 4d). KEGG pathway enrichment analysis revealed significant alterations in seven important pathways, including Staphylococcus aureus infection, Mineral absorption, Biosynthesis of amino acids, Metabolic pathways, Arginine and proline metabolism, Toxoplasmosis, and HTLV-I infection. High-mobility group protein box1 (HMGB1) is a pivotal factor in the development and progression of many types of tumours, which is closely correlated with tumour-mediated inflammation microenvironment [21]. Interestingly, HMGB1 was significantly upregulated after WISP2 overexpression in HCC cells.

\section{Enhanced fibroblast infiltration is related to upregulated HMGB1 in the presence of WISP2 overexpression in $\mathrm{HCC}$}

As determined in the gene expression profiles following WISP2 overexpression in HCC cells, HMGB1 was significantly upregulated, and this upregulation was found to be strongly correlated with cirrhosis in our previous study [21]. In this study, we first validated these gene array assays at the protein level via immunoblotting and confirmed the upregulation of HMGB1 in the presence of WISP2 overexpression (Figure 5A). Next, we generated a Hep3B-WISP2 cell line with stably downregulated expression of HMGB1 (Hep3B-WISP2-shHMGB1; Figure 5B). Hepatic stellate LX2 cells treated with conditioned medium (CM) from Hep3B-WISP2shHMGB1 exhibited inhibited proliferation ability (Figure 5C, 5D). While, LX2 cells treated with CM from Hep3B-WISP2-shHMGB1 exhibited enhanced migration ability $(3.17 \pm 1.17$ vs. $33.83 \pm 12.16, p=$ 0.0001 ; Figure 5E). We then produced subcutaneous tumours in nude mice via transplantation of Hep3BVector, Hep3B-WISP2-Mock, and Hep3B-WISP2shHMGB 1 cells. After 4 weeks, weights of the subcutaneous tumours from Hep3B-WISP2-Vector and Hep3B-WISP2-Mock cells were also not significantly different $(2.78 \pm 0.12 \mathrm{~g}$ vs. $3.02 \pm 0.16$ $\mathrm{g}, p=0.0819)$, while the weights of tumours from Hep3B-WISP2-ShHMGB1 cells were significantly decreased $(0.42 \pm 0.11$ g vs. $2.78 \pm 0.12 \mathrm{~g}, p<0.0001$; Figure 5F). We also found increased expression of $\alpha-$ SMA and fibro-collagen deposition in tumour tissues generated from Hep3B-WISP2-Mock cells, while tumours generated from the Hep3B-WISP2ShHMGB1 cells exhibited significantly decreased $\alpha$-SMA expression and fibro-collagen deposition (Figure 5G). Thus, HMGB1 is involved in the 

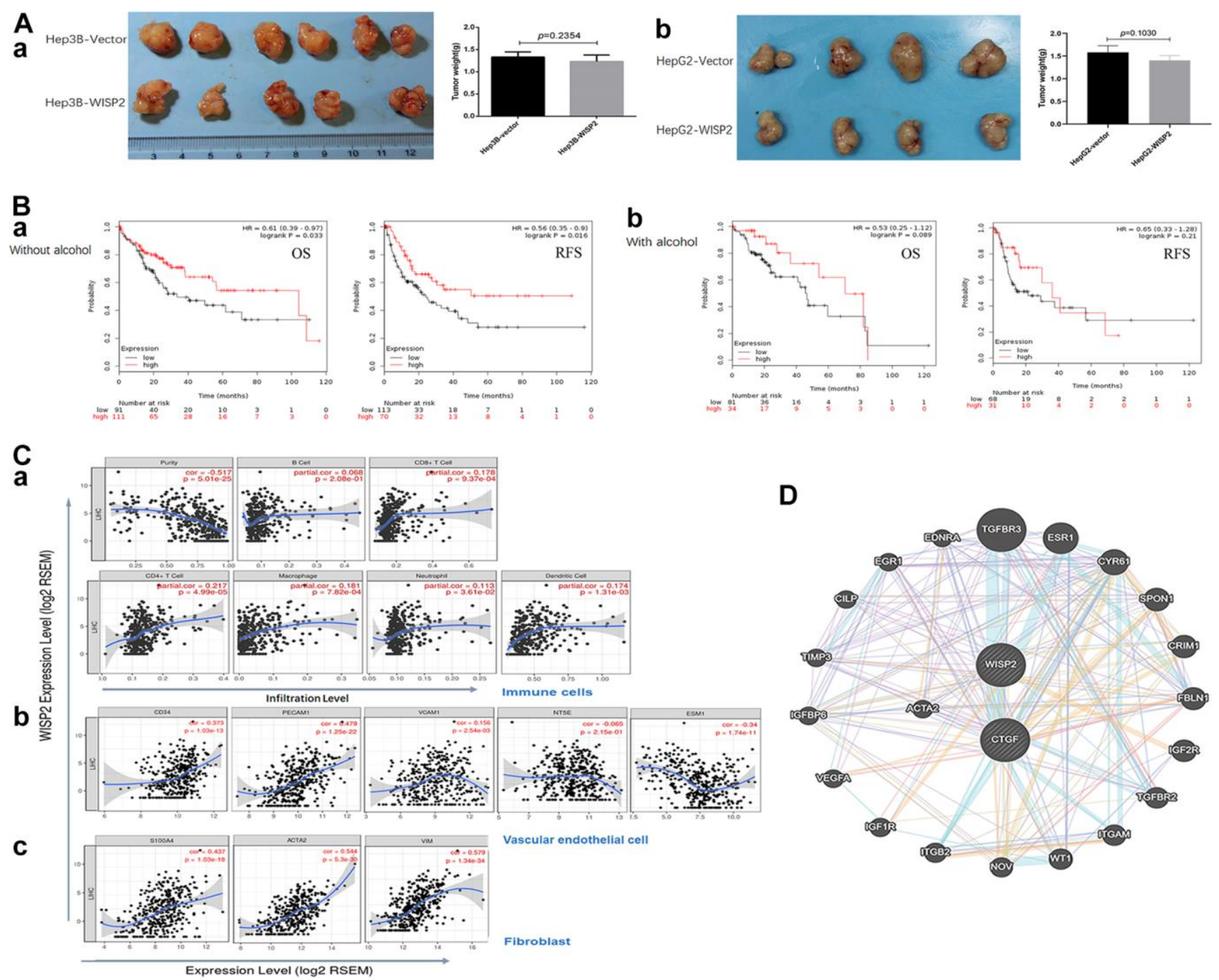

E

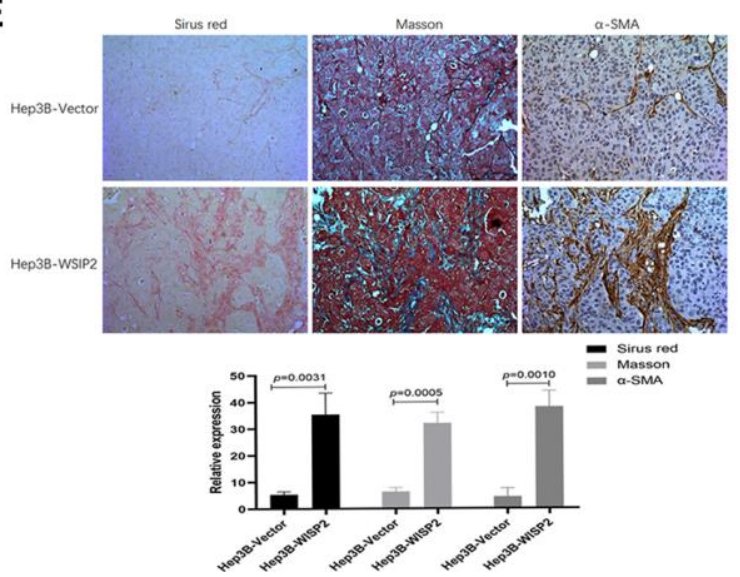

$\mathbf{F}$

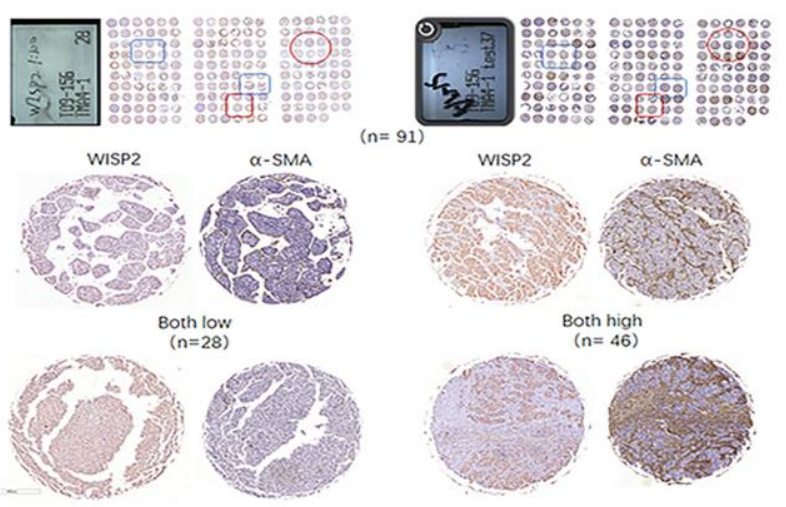

Figure 3. Upregulation of WISP2 is related to tumour purity, and the infiltration of fibroblasts into HCC tissues exerts a negative role in HCC progression. (A, a) In nude mouse models, subcutaneous tumour weights did not differ in mice injected with Hep3BWISP2 and Hep3B-Vector. (A, b) Subcutaneous tumour weights also did not differ in mice injected with HepG2-WISP2 and HepG2-Vector. $(\mathbf{B}, \mathbf{a}, \mathbf{b})$ Higher expression of WISP2 were acting as a protective factor, especially in HCC patients without alcohol intake. (C) WISP2 expression was significantly negatively correlated with tumour purity (a), and was significantly correlated with the specific marker of vascular endothelial cells (b) and fibroblast (c) in TIMER database. (D) The interactions between WISP2 and ACTA2 were evaluated using GeneMANIA database. (E) Increased numbers of fibroblasts and fibro-collagen deposition were positive correlated with the expression of WISP2 in HCC. (F) Human liver cancer tissue microarrays confirmed a positive correlation between WISP2 and $\alpha$-SMA. 
increased fibroblast infiltration that results from WISP2 overexpression (Figure 6).

\section{DISCUSSION}

HCC is the fourth leading cause of cancer death worldwide according to global cancer statistics 2018 [22], and alcohol abuse are considered to be pathogenic factors for HCC in western countries [23, 24]. Although early diagnosis and surgical resection are primary antitumor strategies, the prognosis of cancer patients remains generally dismal, with a 5-year overall survival rate of only $50-70 \%$, and the unfavorable outcomes attributed to the high frequency of tumor recurrence, metastasis, and therapeutic resistance [25]. Therefore, continued identification of new molecules for early survival prediction and the development of molecular targeted therapy are still urgently needed.

Cellular communication network $(\mathrm{CCN})$ family are scaffolding proteins that may govern as localized multitasking signal integrators in tumours and the
A

Hep3B-Vector Hep3B-WISP2

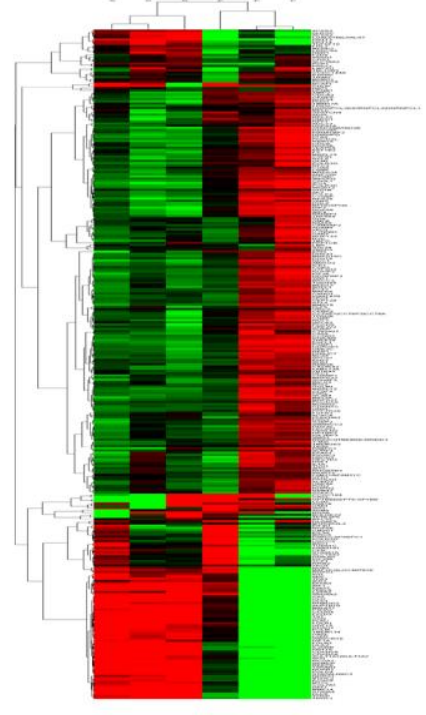

D
B

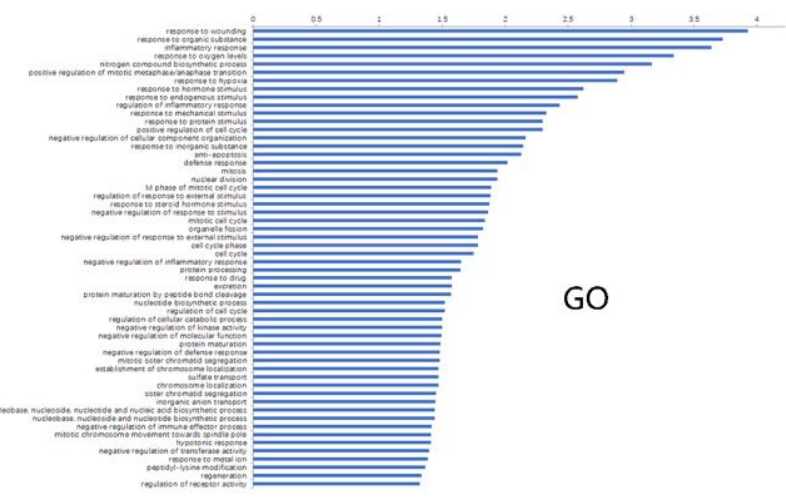

C

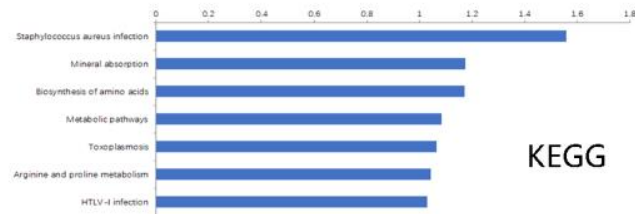

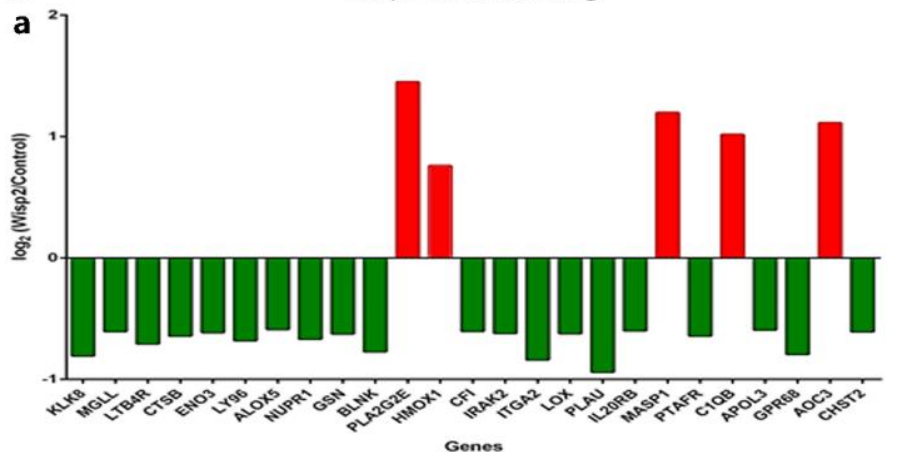
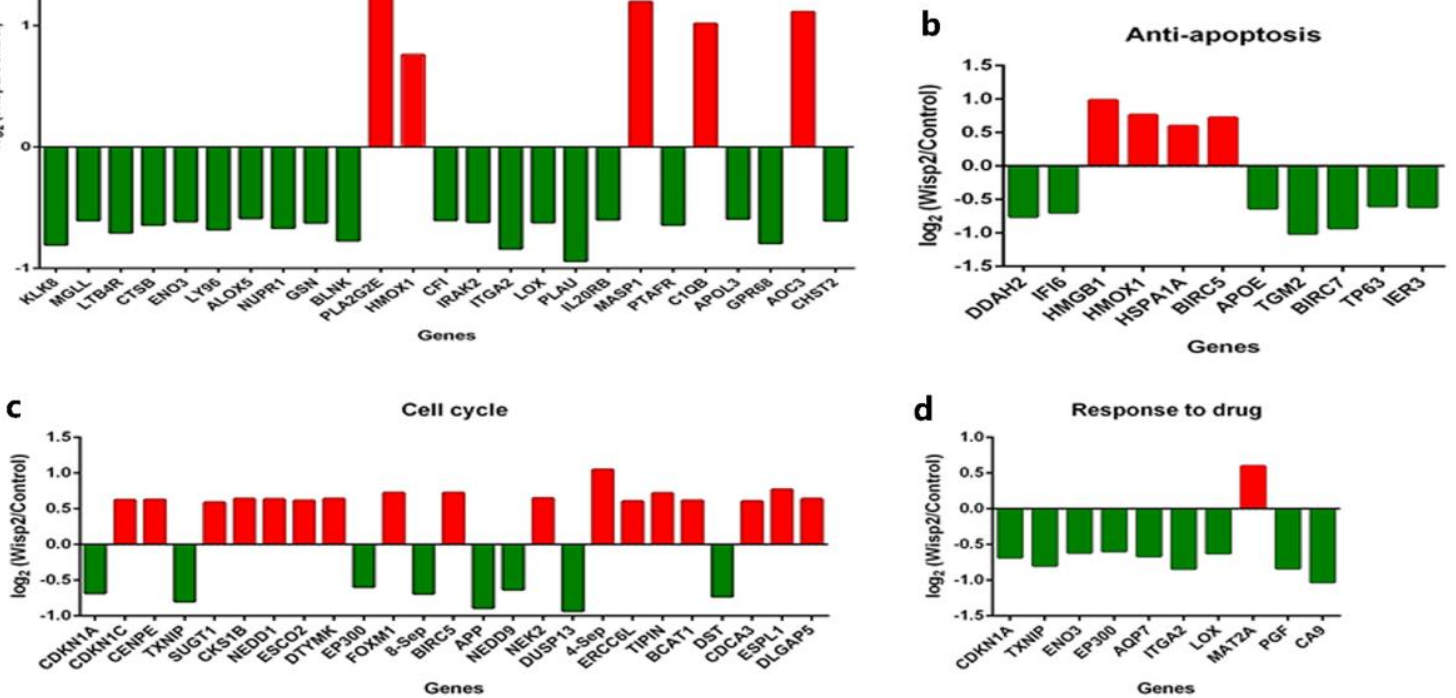

Figure 4. Gene expression profiles were significantly altered in HCC cells that overexpressed WISP2. (A) Heatmap shows the gene expression profiles of Hep3B cells with and without overexpression of WISP2. (B) The differentially expressed genes were evaluated using Gene Ontology analysis. (C) The differentially expressed genes were evaluated by KEGG pathway analysis. (D) Differentially expressed genes were found to be involved in wounding healing (a), anti-apoptosis (b), cell cycle (c), and drug resistance (d). 
associated TME [26]. CCN proteins are involved in many vital biological functions, including angiogenesis, fibrosis, and tissue regeneration and repair [14]. In human cancers, the expression levels of $\mathrm{CCN}$ proteins are closely correlated with in regulating tumor cellular function and the TME [27]. As CCN5 lacks a CT domain, this striking difference in structure compared with other CCN family members may allow it to have unique functional roles. Like its family members, however, previous studies reported inconsistent roles of
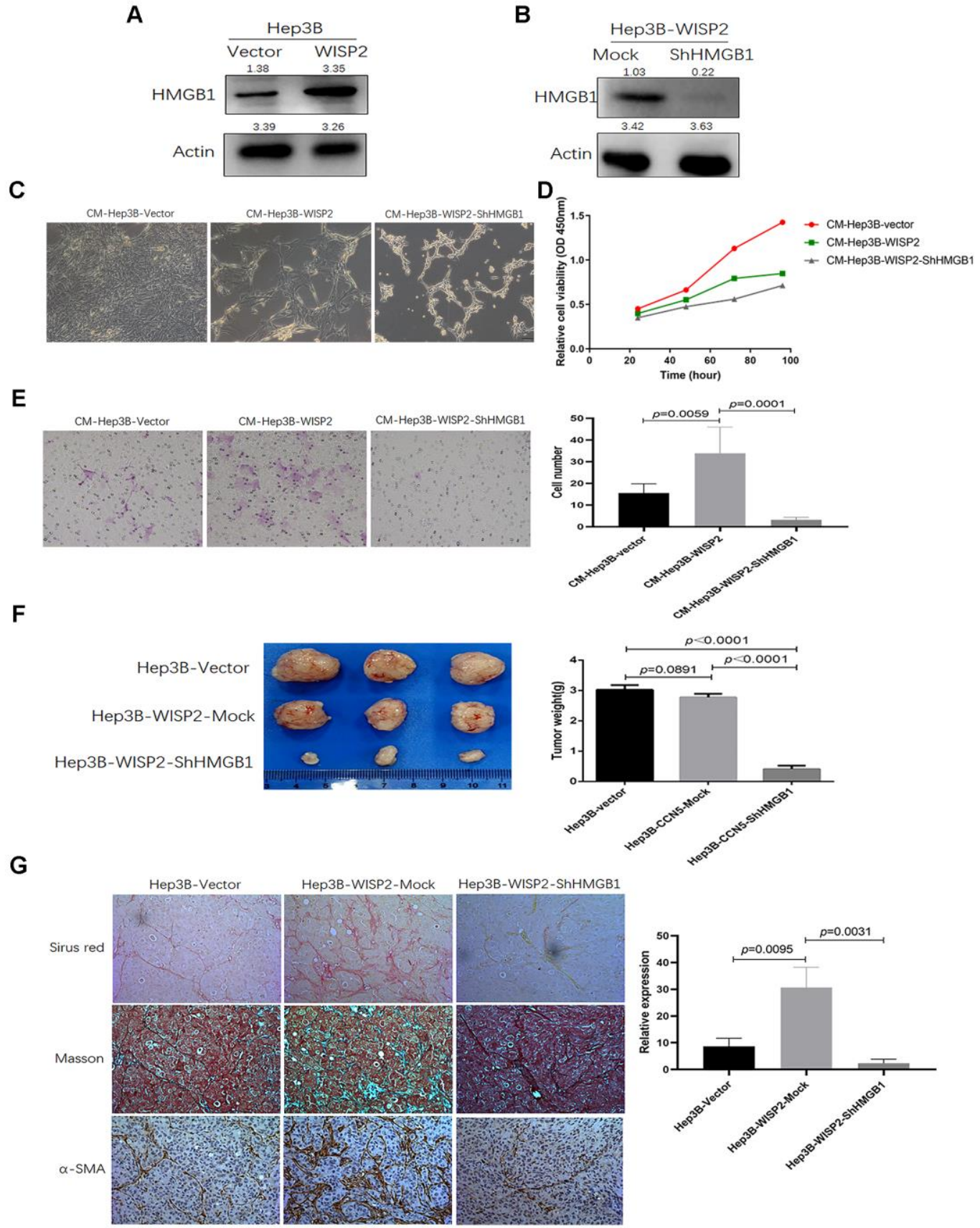

Figure 5. The infiltration of fibroblast cells into HCC tissues is related to HMGB1 after overexpression of WISP2. (A) HMGB1 was significantly upregulated after overexpression of WISP2 at the protein level using immunoblotting in HCC. (B) Immunoblotting was performed to determine the downregulated expression of HMGB1 in Hep3B-WISP2-shHMGB1. (C, D) Hepatic stellate LX2 cells treated with CM from Hep3B-WISP2 or CM from Hep3B-WISP2-shHMGB1, exhibited inhibited proliferation ability. (E) LX2 cells treated with CM from Hep3B-WISP2 exhibited enhanced migration ability, while exhibited inhibited migration ability after treated with CM from Hep3B-WISP2shHMGB1. (F) Subcutaneous tumours in nude mice were induced via inoculation with Hep3B-Vector, Hep3B-WISP2-Mock, and Hep3B-WISP2ShHMGB1 HCC cells, and the weights of tumours from Hep3B-WISP2-ShHMGB1 cells were significantly decreased. (G) Tumours generated from the Hep3B-WISP2-ShHMGB1 cells exhibited significantly decreased $\alpha$-SMA expression and fibro-collagen deposition. 
WISP2 in carcinogenesis. In HCC, the role of WISP2 in tumor progression also remains unclear. Previously, no significant difference in WISP2 expression was identified between HCC tumours and matched normal liver samples [13], indicating that the role of WISP2 in HCC tumour progression remains unresolved. In the present study, data from TIMER and LinkedOmics databases revealed that levels of WISP2 were lower in tumour tissues than in normal liver tissues, and high expression of WISP2 was associated with better prognosis in HCC patients, as revealed by the KaplanMeier plotter, indicating WISP2 is a protective factor in HCC. In addition, in HCC patients without history of alcohol intake, high expression of WISP2 indicated significantly better prognosis, indicating that the effects of WISP2 may be influenced by the microenvironment. In vitro studies also showed that upregulation of WISP2 in HCC is related to inhibition of the malignant phenotype, although such inhibition of proliferation in vivo was not obvious.

Because the in vivo and in vitro studies were inconsistent, we sought to explore potential influences in the tumour microenvironment. Solid tumours are complex entities, as they are surrounded by a heterogeneous array of extracellular matrix and various stromal cells that play important roles in cancer progression. Specifically, tumour-infiltrating lymphocytes are an independent predictor of survival in cancers [28]. In the present study, WISP2 expression was negatively correlated with tumour purity in HCC. And we found WISP2 expression was weakly correlated with infiltrating lymphocytes, including $\mathrm{CD}^{+} \mathrm{T}$ cells, $\mathrm{CD}^{+}{ }^{+} \mathrm{T}$ cells, $\mathrm{B}$ cells, neutrophils, dendritic cells, and macrophages, while WISP2 was positively correlated with the number of fibroblasts in the TME. The co-expression of WISP2 and the fibroblast marker $\alpha$-SMA in subcutaneous tumour tissue and HCC tissue microarrays confirmed this relationship. Therefore, we hypothesized that the inconsistency of results in vitro and in vivo was due to fibroblast infiltration in the TME.

Accumulating evidence supports the concept that cirrhosis is one of the key factors that promotes HCC [29]. And according to the reports, the role of WISP2 appeared to have paradoxical effects in stromal cells. Grünberg et al. [30] showed that WISP2 activates the canonical WNT pathway and increased $\beta$-catenin levels via targeting LRP5/6 phosphorylation in mesenchymal cells. In the myocardium, WISP2 can reverse established cardiac fibrosis via inhibition of enhanced apoptosis of myofibroblasts [31]. In the current study, we demonstrated that $\mathrm{CM}$ from an HCC cell line that was engineered to overexpress WISP2 significantly

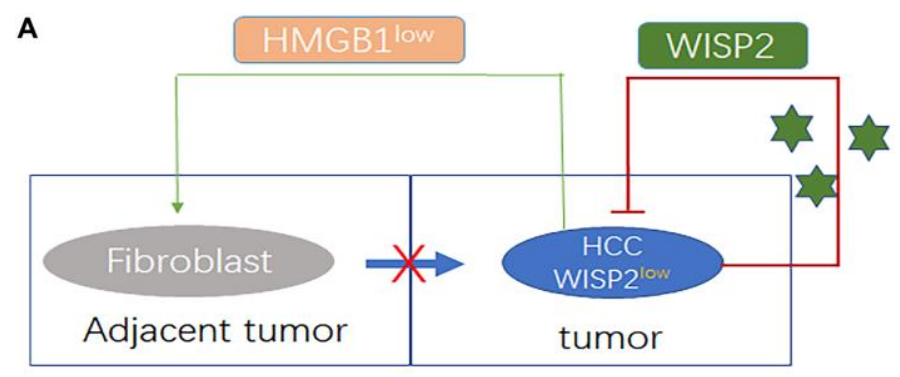

Inhibited malignant feature

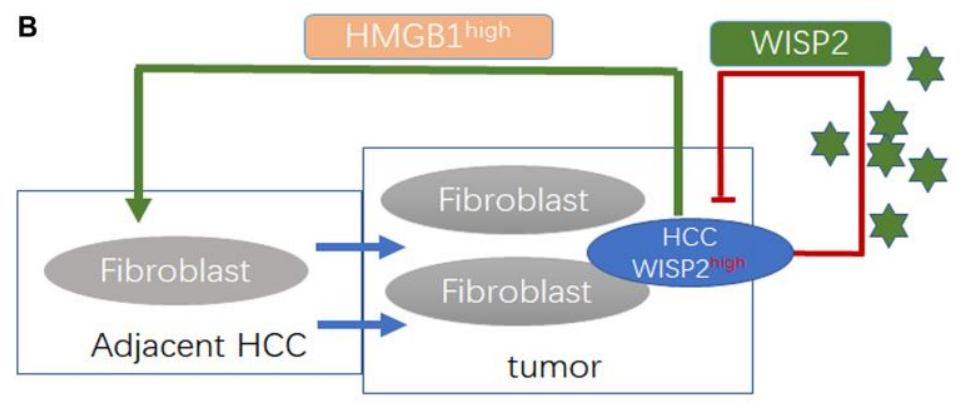

Enhanced malignant feature

Figure 6. WISP2 is a tumour suppressor that is influenced by tumour purity and fibroblast infiltration related to the expression of HMGB1 in HCC. (A) Decreased fibroblast infiltration into HCC tissue was related to the downregulation of HMGB1 in HCC in the presence of low expression of WISP2. Under these conditions, HCC exhibited a restrained state. (B) Increased number of fibroblasts in HCC tissue was related to the upregulation of HMGB1 induced by WISP2. Under these conditions, HCC exhibited a proliferative state. 
inhibited proliferation of LX2, and after downregulation of HMGB1 in these cells, an additional significant decrease in proliferation was observed. While, it is surprised that we proved the CM from an HCC cell line that was engineered to overexpress WISP2 significantly increase the migration ability of LX2, and the trends was reversed in the HCC cell line with stably downregulated expression of HMGB1.

HMGB1 plays a role in several cellular processes, including inflammation, cell differentiation and migration [32]. In our previous study, the upregulation of HMGB1 was found to be strongly correlated with cirrhosis in HCC [21]. According to our screening results, HMGB1 was one of the significantly upregulated genes after WISP2 overexpression in HCC cells, we take HMGB1 as a follow-up research target. In the follow-up study, the weights of subcutaneous tumours generated from these engineered HCC cells in nude mice were also significantly decreased after downregulation of HMGB1 expression in the presence of WISP2 overexpression, indicating that HMGB1 is one of the key factors involved in reducing the anticancer efficiency of WISP2. Additionally, we observed decreased expression of the fibroblast biomarker $\alpha$-SMA in tumour tissues with low expression of HMGB1. These results are in line with previous reports that upregulation of HMGB1 is associated with inflammatory pathogenesis, with enhanced local inflammation and fibrosis [33].

\section{CONCLUSIONS}

We propose that as high expression of WISP2 is associated with better prognosis in HCC, WISP2 can serve as a prognostic biomarker and the prediction efficiency is influenced by tumour purity with fibroblast infiltration. And the enhanced infiltration of fibroblasts is related to upregulated expression of HMGB1, that results from WISP2 overexpression and weakens the anticancer effects of WISP2 (Figure 6). The findings shed light on the dual roles of WISP2 in HCC, suggesting that WISP2 up-regulation combined with HMGB1 inhibition may serve as an effective therapeutic strategy for better prognosis in HCC. While our study was only limited to liver cancer, and lack of deeper mechanistic knowledge of the regulatory relationship between HMGB1 and chemokines from HCC. Therefore, several fundamental questions remain to be answered concerning the mechanism of WISP2 regulating HMGB1 and chemokines in the further study.

\section{Availability of data and material}

The datasets used and/or analyzed and materials developed during the current study are available from the the repository of TCGA (https://portal.gdc.cancer.gov) and corresponding author by reasonable request.

\section{Abbreviations}

AJCC: American Joint Committee on Cancer; CCLE: Cancer Cell Line Encyclopedia; CCN: Cellular Communication Network; CM: Conditioned Medium; GEPIA2: Gene Expression Profiling Interactive Analysis 2; GO: Gene Ontology; HR: Hazard Ratio; HCC: Hepatocellular Carcinoma; HMGB1: HighMobility Group Protein Box1; KEGG: Kyoto Encyclopedia of Genes and Genomes; KIRC: Kidney Renal Clear Cell Carcinoma; KIRP: Kidney Renal Papillary Cell Carcinoma; PPI: Protein-Protein Interaction; TIMER: Tumor IMmune Estimation Resource; TME: Tumor Microenvironment; WISP2: WNT-1-Induced Signalling Protein-2.

\section{AUTHOR CONTRIBUTIONS}

QAJ, YYZ, BHX, XL, YB, ZHX, XLD, and QBZ contributed to the study design, analysis, and interpretation of data. XLD, ZHX, BHX, and YYZ performed the majority of the experiments. YB and XL participated in statistical analysis. QAJ and QBZ drafted and prepared the manuscript. All authors approved the final manuscript.

\section{CONFLICTS OF INTEREST}

The authors declare no conflicts of interest related to this study.

\section{FUNDING}

This research project was mainly supported by the Natural Science Foundation of Shandong Province (ZR2020MH253) and China Postdoctoral Science Foundation funded project (2017M612286). This research project was also partly supported by the National Natural Science Foundation of China (81960533), the Fundamental Research Funds for the Central Universities (31020200QD032), Shaanxi Provincial Innovation Capability Support Program (2019GHJD-14), and Xi'an Science and Technology Action Plan (2019115013YX005SF038(6)).

\section{REFERENCES}

1. Chen W, Zheng R, Baade PD, Zhang S, Zeng H, Bray F, Jemal A, Yu XQ, He J. Cancer statistics in China, 2015. CA Cancer J Clin. 2016; 66:115-32. https://doi.org/10.3322/caac.21338 PMID:26808342 
2. Llovet JM, De Baere T, Kulik L, Haber PK, Greten TF, Meyer T, Lencioni R. Locoregional therapies in the era of molecular and immune treatments for hepatocellular carcinoma. Nat Rev Gastroenterol Hepatol. 2021; 18:293-313.

https://doi.org/10.1038/s41575-020-00395-0 PMID:33510460

3. Jia Q, Xu B, Zhang Y, Ali A, Liao X. CCN Family Proteins in Cancer: Insight Into Their Structures and Coordination Role in Tumor Microenvironment. Front Genet. 2021; 12:649387.

https://doi.org/10.3389/fgene.2021.649387 PMID:33833779

4. Perbal B. CCN proteins are part of a multilayer complex system: a working model. J Cell Commun Signal. 2019; 13:437-39.

https://doi.org/10.1007/s12079-019-00543-5 PMID:31848849

5. Jia $Q$, Dong $Q$, Qin L. CCN: core regulatory proteins in the microenvironment that affect the metastasis of hepatocellular carcinoma? Oncotarget. 2016; 7:1203-14.

https://doi.org/10.18632/oncotarget.6209 PMID:26497214

6. Russo JW, Castellot JJ. CCN5: biology and pathophysiology. J Cell Commun Signal. 2010; 4:119-30.

https://doi.org/10.1007/s12079-010-0098-7 PMID:21063502

7. Banerjee SK, Banerjee S. CCN5/WISP-2: A micromanager of breast cancer progression. J Cell Commun Signal. 2012; 6:63-71.

https://doi.org/10.1007/s12079-012-0158-2 PMID:22487979

8. Davies SR, Watkins G, Mansel RE, Jiang WG. Differential expression and prognostic implications of the CCN family members WISP-1, WISP-2, and WISP-3 in human breast cancer. Ann Surg Oncol. 2007; 14:1909-18. https://doi.org/10.1245/s10434-007-9376-x PMID:17406949

9. Mason HR, Lake AC, Wubben JE, Nowak RA, Castellot JJ Jr. The growth arrest-specific gene CCN5 is deficient in human leiomyomas and inhibits the proliferation and motility of cultured human uterine smooth muscle cells. Mol Hum Reprod. 2004; 10:181-87.

https://doi.org/10.1093/molehr/gah028

PMID:14981145

10. Dhar G, Mehta S, Banerjee S, Gardner A, McCarty BM, Mathur SC, Campbell DR, Kambhampati S, Banerjee SK. Loss of WISP-2/CCN5 signaling in human pancreatic cancer: a potential mechanism for epithelialmesenchymal-transition. Cancer Lett. 2007; 254:63-70. https://doi.org/10.1016/i.canlet.2007.02.012 PMID: 17383817

11. Davies SR, Davies ML, Sanders A, Parr C, Torkington J, Jiang WG. Differential expression of the CCN family member WISP-1, WISP-2 and WISP-3 in human colorectal cancer and the prognostic implications. Int J Oncol. 2010; 36:1129-36. https://doi.org/10.3892/ijo 00000595 PMID:20372786

12. Yang Z, Yang Z, Zou Q, Yuan Y, Li J, Li D, Liang L, Zeng $G$, Chen S. A comparative study of clinicopathological significance, FGFBP1, and WISP-2 expression between squamous cell/adenosquamous carcinomas and adenocarcinoma of the gallbladder. Int J Clin Oncol. 2014; 19:325-35. https://doi.org/10.1007/s10147-013-0550-9 PMID:23592278

13. Zhang $H$, Li W, Huang $P$, Lin $L$, Ye $H$, Lin $D$, Koeffler HP, Wang J, Yin D. Expression of CCN family members correlates with the clinical features of hepatocellular carcinoma. Oncol Rep. 2015; 33:1481-92.

https://doi.org/10.3892/or.2015.3709

PMID:25571929

14. Li T, Fan J, Wang B, Traugh N, Chen Q, Liu JS, Li B, Liu XS. TIMER: A Web Server for Comprehensive Analysis of Tumor-Infiltrating Immune Cells. Cancer Res. 2017; 77:e108-10.

https://doi.org/10.1158/0008-5472.CAN-17-0307 PMID:29092952

15. Tang Z, Kang B, Li C, Chen T, Zhang Z. GEPIA2: an enhanced web server for large-scale expression profiling and interactive analysis. Nucleic Acids Res. 2019; 47:W556-60.

https://doi.org/10.1093/nar/gkz430

PMID:31114875

16. Barretina J, Caponigro G, Stransky N, Venkatesan K, Margolin AA, Kim S, Wilson CJ, Lehár J, Kryukov GV, Sonkin D, Reddy A, Liu M, Murray L, et al. The Cancer Cell Line Encyclopedia enables predictive modelling of anticancer drug sensitivity. Nature. 2012; 483:603-07. https://doi.org/10.1038/nature11003 PMID:22460905

17. Nagy Á, Lánczky A, Menyhárt O, Győrffy B. Validation of miRNA prognostic power in hepatocellular carcinoma using expression data of independent datasets. Sci Rep. 2018; 8:9227.

https://doi.org/10.1038/s41598-018-27521-y PMID:29907753

18. Warde-Farley D, Donaldson SL, Comes O, Zuberi K, Badrawi R, Chao P, Franz M, Grouios C, Kazi F, Lopes CT, Maitland A, Mostafavi S, Montojo J, et al. The GeneMANIA prediction server: biological network 
integration for gene prioritization and predicting gene function. Nucleic Acids Res. 2010; 38:W214-20.

https://doi.org/10.1093/nar/gkq537

PMID:20576703

19. Vasaikar SV, Straub P, Wang J, Zhang B. LinkedOmics: analyzing multi-omics data within and across 32 cancer types. Nucleic Acids Res. 2018; 46:D956-63. https://doi.org/10.1093/nar/gkx1090 PMID:29136207

20. Jepsen P, Kraglund F, West J, Villadsen GE, Sørensen $H T$, Vilstrup H. Risk of hepatocellular carcinoma in Danish outpatients with alcohol-related cirrhosis. J Hepatol. 2020; 73:1030-36.

https://doi.org/10.1016/j.jhep.2020.05.043 PMID: $\underline{32512015}$

21. Zhang $Q B$, Jia $Q A$, Wang $H, H u C X$, Sun $D$, Jiang $R D$, Zhang ZL. High-mobility group protein box1 expression correlates with peritumoral macrophage infiltration and unfavorable prognosis in patients with hepatocellular carcinoma and cirrhosis. BMC Cancer. 2016; 16:880.

https://doi.org/10.1186/s12885-016-2883-z PMID:27836008

22. Bray F, Ferlay J, Soerjomataram I, Siegel RL, Torre LA, Jemal A. Global cancer statistics 2018: GLOBOCAN estimates of incidence and mortality worldwide for 36 cancers in 185 countries. CA Cancer J Clin. 2018; 68:394-24.

https://doi.org/10.3322/caac.21492 PMID:30207593

23. Feng RM, Zong YN, Cao SM, Xu RH. Current cancer situation in China: good or bad news from the 2018 Global Cancer Statistics? Cancer Commun (Lond). 2019; 39:22.

https://doi.org/10.1186/s40880-019-0368-6 PMID:31030667

24. Seitz HK, Bataller R, Cortez-Pinto H, Gao B, Gual A, Lackner C, Mathurin P, Mueller S, Szabo G, Tsukamoto H. Alcoholic liver disease. Nat Rev Dis Primers. 2018; 4:16.

https://doi.org/10.1038/s41572-018-0014-7 PMID:30115921

25. Winkler J, Abisoye-Ogunniyan A, Metcalf KJ, Werb Z. Concepts of extracellular matrix remodelling in tumour progression and metastasis. Nat Commun. 2020; 11:5120.

https://doi.org/10.1038/s41467-020-18794-x

PMID:33037194

26. Yeger H, Perbal B. CCN family of proteins: critical modulators of the tumor cell microenvironment. J Cell Commun Signal. 2016; 10:229-40. https://doi.org/10.1007/s12079-016-0346-6 PMID:27517291

27. Perbal B. The concept of the $\mathrm{CCN}$ protein family revisited: a centralized coordination network. J Cell Commun Signal. 2018; 12:3-12. https://doi.org/10.1007/s12079-018-0455-5 PMID:29470822

28. Quail DF, Joyce JA. Microenvironmental regulation of tumor progression and metastasis. Nat Med. 2013; 19:1423-37.

https://doi.org/10.1038/nm.3394 PMID:24202395

29. Nault JC, Ningarhari M, Rebouissou S, Zucman-Rossi J. The role of telomeres and telomerase in cirrhosis and liver cancer. Nat Rev Gastroenterol Hepatol. 2019; 16:544-58.

https://doi.org/10.1038/s41575-019-0165-3

PMID: $\underline{31253940}$

30. Grünberg JR, Hammarstedt A, Hedjazifar S, Smith U. The Novel Secreted Adipokine WNT1-inducible Signaling Pathway Protein 2 (WISP2) is a Mesenchymal Cell Activator of Canonical WNT. J Biol Chem. 2014; 289:6899-907.

https://doi.org/10.1074/jbc.M113.511964

PMID:24451367

31. Jeong D, Lee MA, Li Y, Yang DK, Kho C, Oh JG, Hong G, Lee $\mathrm{A}$, Song $\mathrm{MH}$, LaRocca TJ, Chen J, Liang L, Mitsuyama S, et al. Matricellular Protein CCN5 Reverses Established Cardiac Fibrosis. J Am Coll Cardiol. 2016; 67:1556-68.

https://doi.org/10.1016/j.jacc.2016.01.030

PMID:27150688

32. Siddiqui SS, Dhar C, Sundaramurthy V, Sasmal A, Yu $H$, Bandala-Sanchez E, Li M, Zhang $X$, Chen $X$, Harrison LC, Xu D, Varki A. Sialoglycan recognition is a common connection linking acidosis, zinc, and HMGB1 in sepsis. Proc Natl Acad Sci U S A. 2021; 118:e2018090118.

https://doi.org/10.1073/pnas.2018090118 PMID: $\underline{3658363}$

33. Zhao J, Yu J, Xu Y, Chen L, Zhou F, Zhai Q, Wu J, Shu B, Qi S. Epidermal HMGB1 Activates Dermal Fibroblasts and Causes Hypertrophic Scar Formation in Reduced Hydration. J Invest Dermatol. 2018; 138:2322-32. https://doi.org/10.1016/j.jid.2018.04.036 PMID:29787749 


\section{SUPPLEMENTARY MATERIALS}

\section{Supplementary Materials and Methods}

\section{Vector construction, transfection, and lentivirus transduction}

Human full-length WISP2 cDNA was obtained from GeneCards (Shanghai, China) and cloned into the pCDH lentiviral expression vector (System Biosciences, Palo Alto, CA, USA). The amplified fragment was inserted into the pCDH plasmid (between $\mathrm{XbaI}$ and EcoRI sites) using the In-Fusion HD Cloning Kit (Takara, Tokyo, Japan). High-mobility group protein 1 (HMGB1) shRNA and scramble plasmids were obtained as a gift from the Institutes of Biomedical Sciences at Fudan University (Shanghai, China), and each was in the lentiviral expression plasmid PLKO.1. Stable cell lines were created by antibiotic selection with puromycin for a week, beginning $72 \mathrm{~h}$ after transduction.

\section{Animal models}

A total of 21 male BALB/c nu/nu mice (aged 4-6 weeks and weighing approximately $20 \mathrm{~g}$ ) and raised in a controlled environment with $25^{\circ} \mathrm{C}$ under standard pathogen-free conditions and a natural light/dark cycle (morning 8:00; afternoon 8:00), and were provided with water and standard diet. Hep3B-Vector, Hep3B-WISP2, HepG2-Vector, HepG2-WISP, and Hep3B-WISP2-Mock, and Hep3B-WISP2-shHMGB1 cells were implanted subcutaneously into the upper left flank region of mice to establish subcutaneous xenografts. Tumour weights were evaluated 4 weeks after the treatments. The intraperitoneal injection of pentobarbital $(5 \mathrm{mg} / \mathrm{kg}$ ) combined with cervical spondylolisthesis was used for euthanasia of the mice after the study. The study protocol was approved by the Medical Experimental Animal Care Commission of Northwest Polytechnical University, and all methods were performed in accordance with the relevant guidelines.

\section{Patients and tissue microarray analysis}

Immunohistochemical analysis of tissue microarrays included 186 samples obtained from HCC patients. Written consent was obtained from patients who received curative resection at the Liver Cancer Institute of Zhongshan Hospital of Fudan University between January 2004 and December 2006. Postsurgical follow-up occurred until December 2013, with a median follow-up period of 63 months (range, 0-110 months). Curative resection was defined as complete resection of tumour nodules with clean post-surgical margins. Histopathological diagnosis was performed according to WHO criteria. Ethical approval was obtained from the Research Ethics Committee of Fudan University.

\section{Antibodies, cell lines and preparation of conditioned media (CM)}

Monoclonal antibodies were used for immunoblot and/or Immunohistochemistry analysis, including WISP2, ki67, $\alpha$-SMA, E-cadherin, and Vimentin (Abcam, Cambridge, MA, USA), HMGB1, Snail, and $\beta$-Actin (Proteintech, Chicago, IL, USA).

The HCC cell line Hep3B and hepatic stellate cell line LX2 were obtained from the Liver Cancer Institute of Fudan University (Shanghai, China). All cell lines were cultured in Dulbecco's Modified Eagle's Media (DMEM; GIBCO, Grand Island, NY, USA) containing $10 \%$ foetal bovine serum (FBS; GIBCO) at $37^{\circ} \mathrm{C}$ in a humidified incubator with $5 \% \mathrm{CO}_{2}$.

The Hep3B-WISP2, Hep3B-Vector, and Hep3BWISP2-shHMGB1 cell lines were plated in $\mathrm{T}_{75}$ flasks $\left(1 \times 10^{6}\right.$ cells $)$. Medium was replaced with $6 \mathrm{~mL}$ of fresh DMEM containing 2\% FBS the next day. After $24 \mathrm{~h}$, $\mathrm{CM}$ were centrifuged at $1,000 \times \mathrm{g}$, and the supernatants were collected and designated CM-Hep3B-WISP2, CMHep3B-Vector, and CM-Hep3B-WISP2-shHMGB1. Finally, CM from these cell lines were used for treating LX2 cells.

\section{Migration and invasion assays}

Migration of Hep3B-WISP2 and Hep3B-Vector were evaluated in Boyden chambers containing membranes with $8.0-\mu \mathrm{m}$ pores in 24 -well plates (Corning, Tewksbury, MA, USA). Cells were seeded into the upper chamber of each well in serum-free DMEM $\left(6 \times 10^{4}\right.$ cells/well $)$. DMEM containing $2 \%$ FBS was added to the lower chamber of each well. After $24 \mathrm{~h}$, cells on the underside of the membrane were stained with Giemsa (Sigma Chemical Company, St. Louis, MO, USA), counted, and photographed at $200 \times$ magnification. Cell invasion assays were performed similarly, except that $80 \mu \mathrm{L}$ Matrigel (BD Biosciences, San Jose, CA, USA) was added to each well $6 \mathrm{~h}$ before cells were seeded onto the membrane.

\section{Proliferation assessment}

To investigate the proliferative effect of WISP2 on HCC cells, Hep3B-WISP2 and Hep3B-Vector $\left(1 \times 10^{3}\right.$ 
cells/well) were plated in 6-well plates and cultured with DMEM containing 10\% FBS. Culture medium was replaced every $3 \mathrm{~d}$, and the colonies were fixed with ice-cold $4 \%$ paraformaldehyde after $14 \mathrm{~d}$. Cells were stained with Giemsa (Sigma, St. Louis, MO, USA) and photographed at $\times 5$ magnification.
Hep3B-WISP2 and Hep3B-Vector were plated in 96-well plates $\left(3 \times 10^{3}\right.$ cells/well $)$ and cultured for 24 , 48, 72, and $96 \mathrm{~h}$. Cell proliferation assays were carried out with the Cell Counting Kit 8 (CCK8; Dojindo). Results were expressed as the absorbance of each well at $450 \mathrm{~nm}(\mathrm{OD} 450)$. 